\title{
Correction of Spherical Aberration and Six-Fold Astigmatism Using Three Dodecapoles
}

\author{
H. Sawada ${ }^{*, * *}$, T. Sasaki ${ }^{*, * *}$, F. Hosokawa**, S. Yuasa, M. Terao ${ }^{* *}$, M. Kawazoe $^{* *}$, K. Omoto $^{* * * *},{ }^{* * *}$, T.

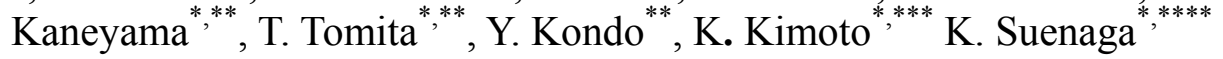

*Japan Science and Technology Agency, CREST, 5, Sanbancho, Chiyoda-ku, Tokyo, 102-0075, Japan

** JEOL Ltd., 3-1-2 Musashino, Akishima, Tokyo, 196-8558, Japan

${ }_{* * * *}^{* *}$ National Institute for Materials Science (NIMS), 1-1 Namiki, Tsukukba, Ibaraki 305-0044, Japan

${ }^{* * * *}$ National Institute of Advanced Industrial Science and Technology (AIST), Tsukuba, 305-8565, Japan

Since multipole electron optics systems for aberration correction have been established, the aberration correctors overcome limitations caused by spherical aberration (Cs) [1-4]. In particular, double hexapole type Cs correctors, which were firstly developed by H. Rose and M. Haider [1, 2], are widely used for an electron microscopy. The corrector can correct residual aberration up to the fourth order as well as Cs. However, aberrations higher than fifth order are not completely adjusted in the conventional system. In the system, a six-fold astigmatism (A6) dominantly remains among residual aberrations and limits higher resolution. To compensate the A6, P. Hartel et al. proposed and made a system with asymmetrical of double dodecapole [5]. However, it is important to explore the possibility to compensate it by other ways and to evaluate to find the best way. We propose a new Cs correction system of triple dodecapole (so called the delta system: Dodecapole Element Triple Aberration Corrector) to compensate A6, which is designed and actually tested based on the calculated prediction by us. This paper reports the principle and experiment of the system. In this paper, we only state for STEM configuration, because it is equivalent to TEM, which is mirror-symmetric one of the STEM.

In low-acceleration-voltage high-resolution electron microscopy [6], the correction of A6 is especially important. A lager convergent angle to form a probe is required to resolve atomic scale objects, because of the longer wavelength of the electrons, resulting in a larger diffraction limit $(0.61 \lambda / \alpha$ for STEM).

In the delta system, the Cs from objective lens is compensated by summation of three hexaploe field from three dodecapoles; similar to the conventional hexapole system $[1,2]$. The impeditive three-fold astigmatism (A3) is compensated as a sum of three vectors of hexapole fields generated by the dodecapoles. That is, the azimuth direction of A3 generated in the first dodecapole is rotated at the second one and the A3 is finally compensated at the third one [Fig.1]. The A6 mainly generated a cross term derived from mutual relation of two hexapole fields. The directions and amplitudes of the A6s from two of three dodecapoles are tuned so that the total A6 are compensated. Optimum inter-rotation angles between three hexapole fields and the amplitudes of those are derived from the theoretical calculation. The delta system was tested with an actual scanning transmission electron microscope at $60-\mathrm{kV}$ acceleration. Experimental result showed compensation of a A6 as well as Cs resulting in the roughly double coherently convergent angle compared to one for double hexapoles [Fig.2 and a report by Sasaki et al [7]].

This work is supported by the CREST project under Japan Science and Technology agency (JST). 
[1] H. Rose, Optik 85, 19 (1990)

[2] M. Haider et al, Nature 392, 768 (1998).

[3] O. L. Krivanek, M. Dellby, and A. R. Lupini, Ultramicrosc. 78, 1(1999)

[4] F. Hosokawa et al, IMC 16, 582 (2006),

[5] P. Hartel et al, Microsc. Microanal. 13 Suppl. 2, 1148 (2007).

[6] K. Suenaga, to be published.

[7] T. Sasaki et al, in this congress.

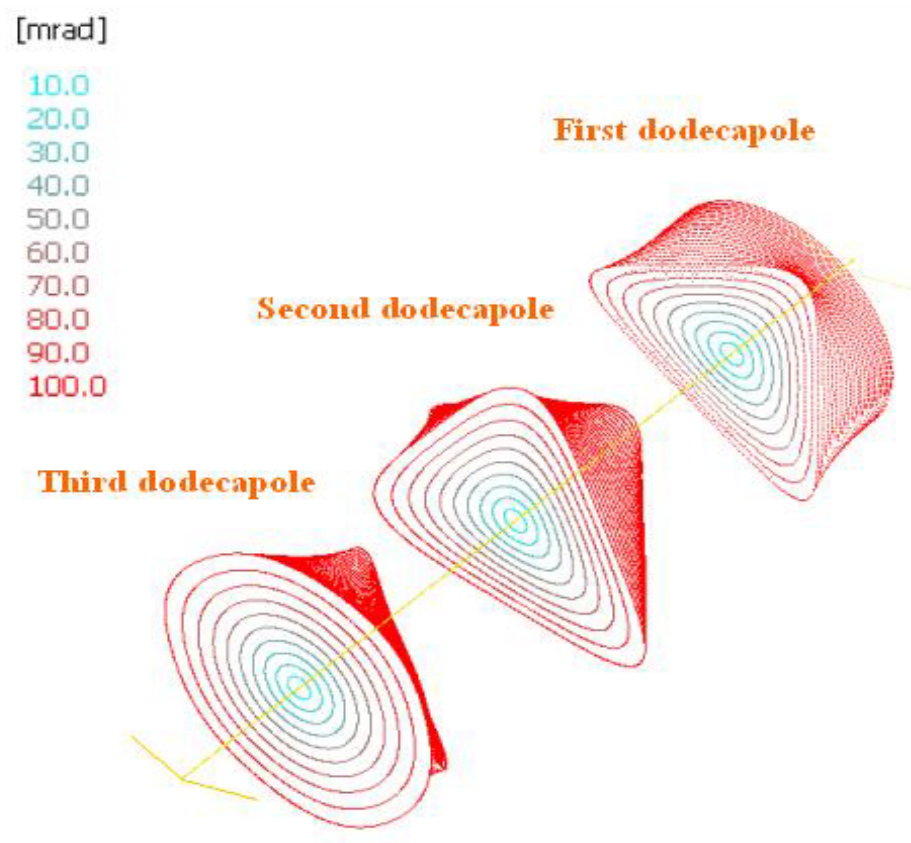

\section{Figure 1}

Calculated trajectory of triple dodecapole system.

The pattern like annual growth rings shows the cross-section of the beam. Each of the contours corresponds to the convergent semi-angle on a specimen, which is listed in upper left of the figure. Note that only the electron trajectories in the dodecapoles are shown, whereas that in transfer lens system is hidden to show the cross section in this figure.

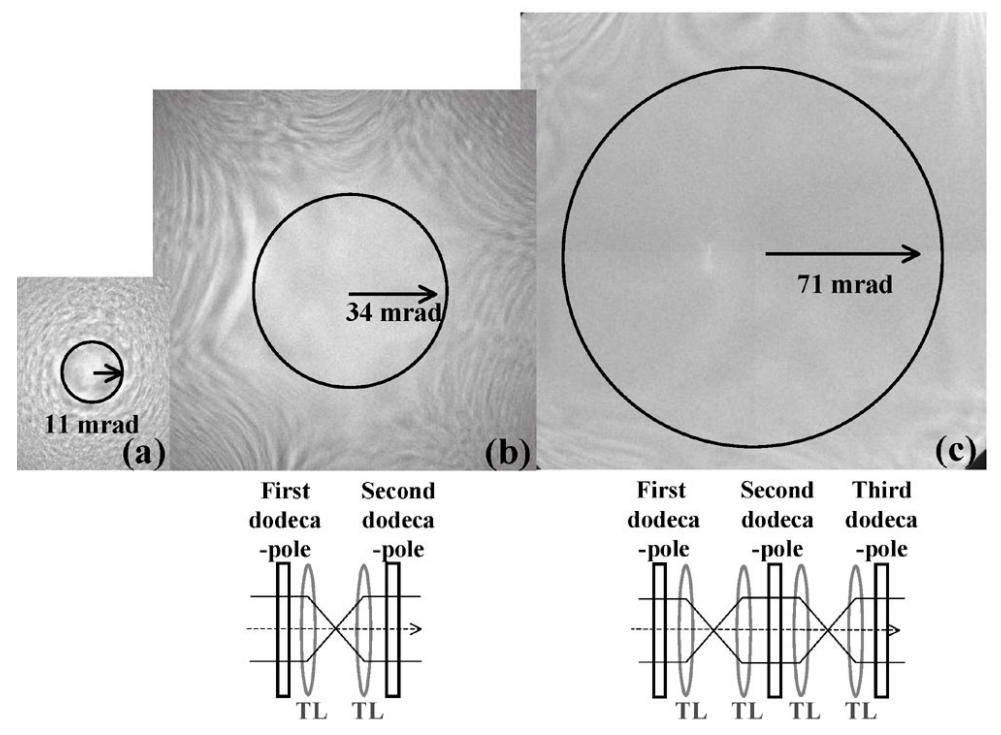

Figure 2

Ronchigrams for STEM;

(a) Non-corrected.

(b) Corrected by double dodecapoles.

(c) Corrected by triple dodecapoles.

Axial ray paths of correction systems are shown in the lower panel of each Ronchigram. The observed infinity magnification area are 11, 34, 71 mrad of semi-angle for (a), (b) and (c), respectively. 